\title{
A Systematic Review on the Sufficiency of PubMed and Google Scholar for Biosciences
}

\author{
Yun Hwee Teo ${ }^{1}$ and Maurice HT Ling ${ }^{1,2 *}$ \\ ${ }^{1}$ School of Applied Sciences, Temasek Polytechnic, Singapore \\ ${ }^{2}$ HOHY PTE LTD, Singapore \\ *Corresponding Author: Maurice HT Ling, School of Applied Sciences, Temasek \\ Polytechnic and HOHY PTE LTD, Singapore.
}

Received: September 23, 2020

Published: November 10, 2020

(C) All rights are reserved by Yun Hwee Teo and

Maurice HT Ling.

\begin{abstract}
NCBI PubMed is the de facto bibliographic database for biosciences but has been shown to be insufficient for the purpose of systematic review and meta-analysis, which requires comprehensiveness. Among bibliographic databases, Google Scholar is most comprehensive. With arguments that PubMed, supplemented with Google Scholar, may be sufficient for a systematic review in biosciences; we reviewed 18 studies to determine whether a combination of PubMed and Google Scholar is sufficient. Current literature shows that the combined coverage of Google Scholar and PubMed is between $85 \%$ to $98 \%$ of the universe of bioscience articles, which may be sufficient. However, Google Scholar alone is not sufficient as the concordance between PubMed and Google Scholar is $30.3 \%$ with $20.3 \%$ of the articles unique to PubMed.
\end{abstract}

Keywords: Systematic Review; PubMed; Google Scholar

\section{Introduction}

In the field of biosciences, NCBI PubMed is the de facto bibliographic database for literature published after 1950 [1] and it has been growing at the rate of about 4\% from 1986 to 2010 [2]. There is little coverage of pre-1950 publications. More importantly, a study in 2015 suggests that PubMed may not be comprehensive [3]. Hence, PubMed alone is insufficient for the purpose of systematic review and meta-analysis [4], which requires comprehensiveness $[5,6]$. As such, there are substantial studies on the combinations of bibliographic databases to search for a systematic review in biosciences.

Using frozen shoulder management as a case study, Beyer and Wright [7] suggest that at least two databases are required. In 2016, Bramer., et al. [8] suggest that a combination of Embase, PubMed/MEDLINE, and Google Scholar may be required as both individual databases and a combination of Embase and PubMed do not provide sufficient coverage. A follow-up study in 2017 [9] examined 8 citation databases and conclude that a combination of 4 databases; namely, Embase, PubMed, Web of Science Core Collection, and Google Scholar; as the minimum to achieve sufficient coverage. However, both Web of Science and Embase require subscription. Hence, the question of whether the combination of PubMed and Google Scholar is sufficient for a systematic review in biosciences, remains.

Recently in 2019, Michael Gusenbauer [10] examined the sizes of 12 academic search engines and bibliographic databases and concludes that Google Scholar is the most comprehensive. Google Scholar is known for its coverage of grey literature [11], a collective term referring to published written material that not indexed by major database vendors; which can include white papers, technical reports, standard documents, specifications, conference proceedings, and even graduate dissertations. However, Haddaway., et al. [12] caution against the use of Google Scholar alone for systematic review searches while echoing its application in finding grey literature. These studies suggest that Google Scholar should be used in tandem with a standard bibliographic database in the field of study. 
Indeed, there are arguments that PubMed, supplemented with Google Scholar/Google, may be sufficient coverage for systematic reviews [13].

Here, we review 18 studies to determine whether a combination of PubMed and Google Scholar is sufficient for a systematic review in biosciences. With the combined coverage of Google Scholar and PubMed between $85 \%$ to $98 \%$ of the universe of bioscience articles, it may be sufficient. However, Google Scholar alone is not sufficient as $20.3 \%$ of the articles may be unique to PubMed.

\section{Method}

\section{Search strategy}

A literature search was undertaken on July 28, 2020, to locate studies examining the sufficiency of PubMed (https://pubmed. ncbi.nlm.nih.gov) and Google Scholar (https://scholar.google. com) as search engines for biosciences. Citations from PubMed were searched using both "google" and "pubmed" in title field as "google[ti] AND pubmed[ti]"1. Citations from Google Scholar were searched by requiring both "google scholar" and "pubmed" in title as "allintitle: 'google scholar' pubmed", sort by relevance while excluding patents and citations ${ }^{2}$. Citations from PubMed and Google Scholar were denoted as Set PM and Set GS, respectively.

\section{Inclusion and exclusion criteria}

The following seven exclusion criteria were applied: (A) Within Set PM and Set GS individually, duplicated citations based on title and author names were removed, resulting in unique citations within each set; (B) citations with no access to full-text articles were removed, resulting in only full-text articles; (C) articles that were not written in either English or Chinese language were removed; (D) articles with no mention of both "Google" and "PubMed" within the main text were removed; (E) the resulting Set PM and Set GS were combined and duplicated articles based on title and author names were removed; (F) articles that were not published in journals or conferences were removed; and (G) articles without qualitative nor quantitative comparison(s) between Google Scholar and PubMed in the main text. All articles after exclusion were included for this review.

\section{Results and Discussion}

\section{Analysis of search results}

A hit is defined as an atomic result from a search and the number of hits from a search refers to the number of results returned [14]. 37 hits were returned from Google Scholar (Set GS); of which, 34 were unique with 3 duplicates (Figure 1). The reason for duplication in Google Scholar is that the same publication may be indexed by more than one database. For example, Mastrangelo., et al. [15] is indexed by SpringerLink (https://link.springer.com) and Academia (https://www.academia.edu). 19 hits were returned from PubMed (Set PM). The number of hits from both Google Scholar (Set GS) and PubMed (Set PM) remained constant up to September 20, 2020 (the date of latest check). 51 hits are non-duplicates within the set (Exclusion Criterion A); of which, 42 have full-text articles (Exclusion Criterion B). Within the full-text articles, 38 articles are either in English or Chinese (Exclusion Criterion C); of which, 35 mention "Google" and "PubMed" in the text (Exclusion Criterion D). This resulted in 26 are unique articles (Exclusion Criterion E); of which, 22 articles are journal or conference articles (Exclusion Criterion F). Finally, 18 articles contain comparison(s) between Google Scholar and PubMed (Exclusion Criterion G), which are used in this review.

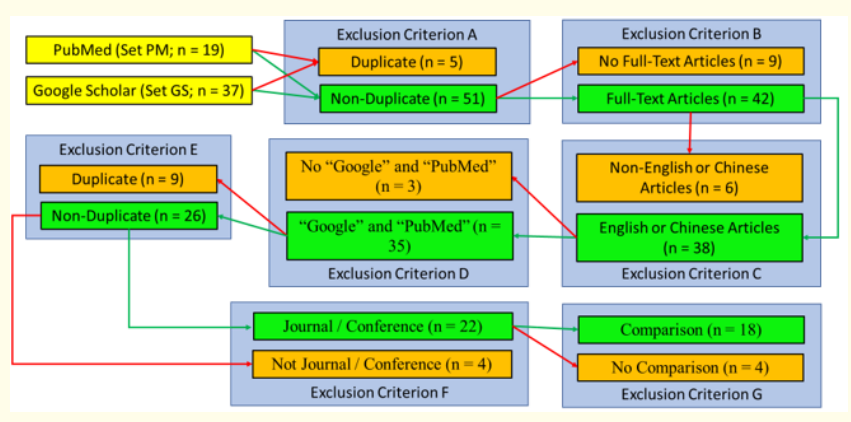

Figure 1: Flow of hits through exclusion criteria.

\section{Are PubMed and Google scholar sufficient?}

PubMed [16] and Google Scholar [10] are probably the most used academic/bibliographical databases in biosciences as they can be accessed without charge [15] and there are arguments [13]

\footnotetext{
${ }^{1}$ https://pubmed.ncbi.nlm.nih.gov/?term=google[ti]+AND+pubmed[ti]

${ }^{2}$ https://scholar.google.com/scholar?q=allintitle:"google+scholar" +pubmed\&hl=en\&as_sdt=1,5\&as_vis=1
} 
that PubMed, supplemented with Google Scholar/Google, may be sufficient coverage for systematic reviews. However, is the combination of PubMed and Google Scholar sufficient for a systematic review in biosciences? Here, we systematically review 18 articles to answer this question. The main findings, in order of year of publication, are as follow:

1. In 2007, Schulz [17] analyzes 10 searches from both PubMed and Google Scholar. Combining the 10 searches, Google Scholar and PubMed returned 247 and 147 hits respectively; of which, 122 hits were common. Schulz also noted that Google Scholar returns hit from journals that are not indexed by PubMed and publications older than 1950.

2. In 2008, Falagas., et al. [16] provide a qualitative comparison focusing on citation analysis and found that results from Google Scholar can be inconsistent compared to PubMed. However, Google Scholar, can help in the retrieval of obscure sources of information.

3. In 2008, Teng [18] notes that Google Scholar returns substantially more hits than PubMed (982 hits from PubMed vs 2740 hits from Google Scholar). However, the searches are not directly comparable as PubMed searches uses search tags for journal names ("TA" tag) and author affiliations ("AD" tag), which do not have an equivalent in Google Scholar. Teng also notes that despite an easy search interface, Google Scholar is limited in modifying search terms and gave an example difficulty in finding journal articles and conference articles separately.

4. In 2008, Xue., et al. [19] analyze 10 searches from both PubMed and Google Scholar, which is similar to the protocol undertaken by Schulz [17]. It is interesting that Xue., et al. [19] report identical results to Schulz [17] (Table 2-4 are identical in both publications). However, Schulz did not provide the exact search terms in her publication whereas Xue., et al. [19] present their search terms in table 1 of their publication. Table 1 of Schulz [17] is a summary of criticisms against Google Scholar.

5. In 2009, Freeman., et al. [20] compare the search results from three drug information specialists across two days and found that Google Scholar returned significantly more hits than PubMed (2211.6 hits from Google Scholar vs 44.2 hits from PubMed; p-value $=0.019$ ). However, more primary literature articles published pre-2000 were in Google Scholar hits compared to PubMed (62.8\% vs 34.9\%; p-value $=0.017$ ) but the differences for the number of articles published post2000 were not significant (66.4 vs 77.1; p-value $=0.074$ ).
6. In 2010, Wang [21] examines the search hit using "brucellosis" as search term for Google Scholar and PubMed, but did not present statistics of the search. However, Wang notes that PubMed has a false positive rate of about $5 \%$ and Google Scholar can locate earlier publications.

7. In 2010, Anders and Evans [22] perform three clinically relevant searches and show that Google Scholar return substantially more hits than PubMed (80730 hits from Google Scholar vs 467 hits from Google PubMed). However, the recall for both PubMed and Google Scholar are similar but PubMed has better precision (p-value < 0.001).

8. In 2010, Mastrangelo., et al. [15] examine papers related to sarcoma risk factors using Google Scholar and PubMed and reported substantially more hits from Google Scholar due to search filtration limitations. After filtering their search manually, their result shows 74 hits from Google Scholar against 46 hits from PubMed. Of which, 9 hits are common.

9. In 2010, Hightower and Caldwell [23] survey 220 staffs and students using the science and engineering departmental email lists at the University of California Santa Cruz to identify preferences of database use. They found statistically similar preferences for PubMed (21.5\%) and Google Scholar (18.7\%).

10. In 2011, Tober [24] evaluates the search results using "multiphoton microscopy" as search term in both Google Scholar and PubMed. Google Scholar returns 29000 hits while PubMed returns 1693 hits. However, it is surprising that Google Scholar gives higher precision than PubMed (mean precision of $25 \%$ from Google Scholar compared to 20\% from PubMed).

11. In 2012, Nourbakhsh., et al. [25] compares the search results from Google Scholar and PubMed using four searches. Google Scholar returns 64 hits while PubMed returns 50 hits; of which, 13 hits are common. 67.6\% of PubMed articles and $80 \%$ of Google Scholar articles are considered relevant, which can be indicative of recall.

12. In 2013, Perryman [26] presents a commentary on the work of Nourbakhsh., et al. [25]; hence, does not present new results.

13. In 2013, Shariff., et al. [27] examine the search results by finding nephrological review. Using the first 40 hits, they found that Google Scholar returns twice as many relevant articles (11\% from PubMed and 22\% from Google Scholar; p-value < $0.001)$ compared to PubMed, but precision was similar $(6 \%$ precision from PubMed and 8\% precision from Google Scholar; -value $=0.07$ ). Moreover, Google Scholar provides greater access to free full-text publications ( $5 \%$ of PubMed citations 
compared to $14 \%$ of Google Scholar article; p-value < 0.001). In terms of coverage, both contained $78 \%$ of the 1574 unique citations collected. Google Scholar contained an additional 5\% of the articles not included in PubMed and PubMed contained an additional $2 \%$ of the articles not included in Google Scholar. Yet, $15 \%$ of the articles were missing in both sources.

14. In 2013, Bramer., et al. [28] identify 21 systematic reviews and using the reviewed articles in these 21 systematic reviews as items to search, giving a total of 541 articles, in order to compare Google Scholar and PubMed search results. Using comparable searches, PubMed returns 369 relevant hits (68\% recall) while Google Scholar returns 389 relevant hits (72\% recall) within the first 1000 hits. Importantly, Bramer., et al. report 531 out of 541 articles can be found using Google Scholar (98\% coverage) whereas only 493 out of 541 articles can be found using PubMed (91\% coverage).

15. In 2014, Wakimoto [29] presents a commentary on the work of Shariff., et al. [27]; hence, does not present new results.

16. In 2015, Badgett., et al. [30] use a set of 312 searches to the search results of Google Scholar and PubMed and found that $65 \%$ of PubMed searches and $20 \%$ of Google Scholar searches return at least one citation.

17. In 2020, Gusenbauer and Haddaway [31] evaluate the multiple search engines qualitatively, including Google Scholar and PubMed, for their usability in conducting systematic reviews and meta-analyses. Hence, does not provide quantitative data for comparison between Google Scholar and PubMed. However, the authors note that the coverage of Google Scholar is substantially larger than PubMed. In fact, Google Scholar has the largest coverage of more than 389 million articles among the 33 academic search engines reviewed.

18. In 2020, Morshed and Hayden [32] examines the search results from Google Scholar and PubMed on two clinical questions. In total, Google Scholar returns 262 hits with 35\% relevance while PubMed returns 194 hits with 29\% relevance.
Taking these 18 articles together, it is clear that Google Scholar returns substantially more hits than PubMed - as much as 173 times more hits has been observed [22]. This can be attributed to the coverage of Google Scholar [31]. Coverage is the most crucial measure in determining whether a combination of Google Scholar and PubMed is sufficient. With the combined coverage of Google Scholar and PubMed between 85\% [27] to 98\% [28] of the universe of articles, it may be sufficient.

\section{Is Google Scholar alone sufficient?}

Given that several studies elucidated that Google Scholar is likely the most comprehensive academic/bibliographical database in terms of volume and coverage $[10,33]$, it leads to wonder whether searching Google Scholar alone is sufficient [8] despite a small percentage of articles unique to PubMed [27]. A side effect of this study has indirectly offered us an answer to this question. Our analysis of the 51 non-duplicate hits after exclusion criterion A (Figure 2) shows that the 51 hits originate from 32 hits in Set GS and 19 hits in Set PM. Only 10 hits are common in both Set GS and Set PM giving a concordance measure [34] of 24.4\%. More importantly, 3 of the 18 reviewed articles [25,30,32] are only found in Set PM. Combining our results with that of other studies (Table 1), a concordance of $30.3 \%$ is obtained with 102 (20.3\%) articles unique to PubMed. These results suggest that Google Scholar is not a superset of PubMed; hence, Google Scholar alone is insufficient.

Furthermore, our analysis of set GS uncovered an interesting phenomenon - the initial search results showed 38 hits which spanned across 4 pages; however, the number of hits changed from 38 to 37 between page 2 and 3 (Figure 3). A study by Xing., et al. [35] demonstrated that hits returned from Google searches can differ based on geographical location. Taken together, these support our concordance result that searching Google Scholar alone is not likely to be sufficient.

\begin{tabular}{|l|c|c|c|c|}
\hline Reference & Google Scholar Only & Google Scholar and PubMed & PubMed Only & Concordance Measure (34) \\
\hline$(15)$ & 65 & 9 & 37 & $8.1 \%$ \\
\hline$(17)$ & 125 & 122 & 25 & $44.9 \%$ \\
\hline$(25)$ & 51 & 13 & 37 & $12.9 \%$ \\
\hline This study & 7 & 8 & 3 & $44.4 \%$ \\
\hline Total & 248 & 152 & 102 & $30.3 \%$ \\
\hline
\end{tabular}

Table 1: Concordance of Google Scholar and PubMed. 

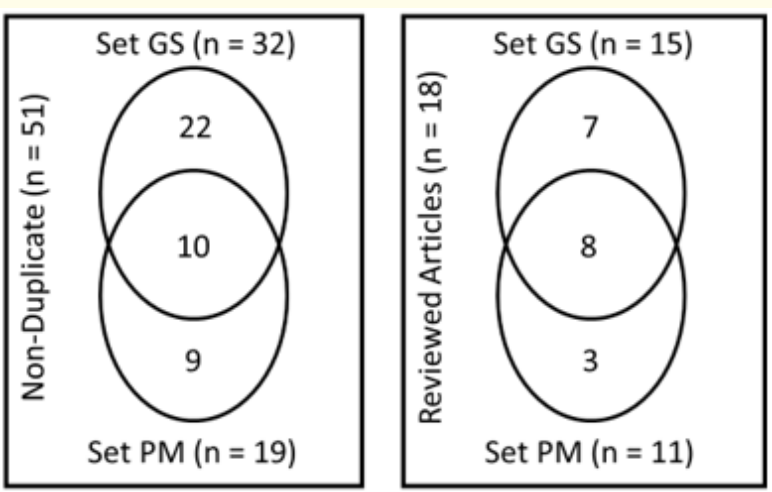

Figure 2: Distribution of 51 non-duplicate hits (After Exclusion Criterion A) and the 18 hits reviewed in this Study within Set GS and Set PM. The concordance measures (34) of non-duplicate hits and reviewed articles are $24.4 \%$ and $44.4 \%$ respectively.

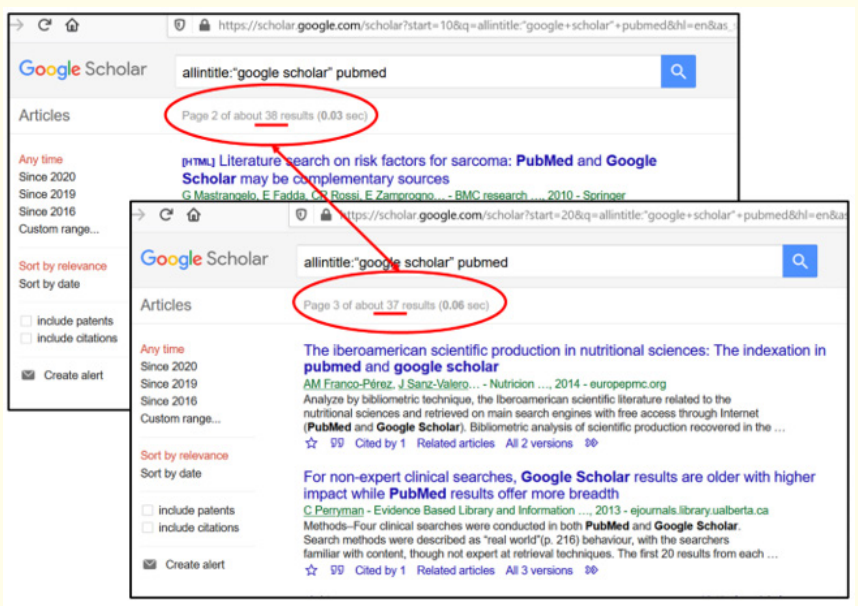

Figure 3: Differences in search results from google scholar during pagination.

\section{Conclusion}

Current literature suggests that combination of PubMed and Google Scholar is sufficient for biosciences with a combined coverage between $85 \%$ to $98 \%$ of the universe of bioscience articles. However, $20.3 \%$ of the articles may be unique to PubMed, suggesting that Google Scholar alone is insufficient.

\section{Conflict of Interest}

The authors declare no conflict of interest.

\section{Bibliography}

1. Kelly L and St Pierre-Hansen N. "So Many Databases, Such Little Clarity: Searching the Literature for the Topic Aboriginal". Canadian Family Physician 54.11 (2008): 1572-1573.

2. Lu Z. "PubMed and Beyond: A Survey of Web Tools for Searching Biomedical Literature". Database Oxford (2011): baq036.

3. Liljekvist MS., et al. "For 481 Biomedical Open Access Journals, Articles are not Searchable in the Directory of Open Access Journals nor in Conventional Biomedical Databases". PeerJ 3 (2015): e972.

4. Zheng B., et al. "Are PubMed Alone and English Literature Only Enough for a Meta-Analysis?" Annals of Oncology 24.4 (2013): 1130.

5. Greyson D., et al. "Systematic Review Searches Must Be Systematic, Comprehensive, and Transparent: A Critique of Perman et al'. BMC Public Health 19.1 (2019): 153.

6. Atkinson LZ and Cipriani A. "How to Carry Out a Literature Search for a Systematic Review: A Practical Guide". BJPsych Advances 24.2 (2018): 74-82.

7. Beyer FR and Wright K. "Can We Prioritise Which Databases to Search? A Case Study Using a Systematic Review of Frozen Shoulder Management". Health Information and Libraries Journal 30.1 (2013): 49-58.

8. Bramer WM., et al. "Comparing the Coverage, Recall, and Precision of Searches for 120 Systematic Reviews in Embase, MEDLINE, and Google Scholar: A Prospective Study". Systematic Reviews 5 (2016): 39.

9. Bramer WM., et al. "Optimal Database Combinations for Literature Searches in Systematic Reviews: A Prospective Exploratory Study". Systematic Reviews 6.1 (2017): 245.

10. Gusenbauer M. "Google Scholar to Overshadow Them All? Comparing the Sizes of 12 Academic Search Engines and Bibliographic Databases". Scientometrics 118.1 (2019): 177-214.

11. Yasin A., et al. "On Using Grey Literature and Google Scholar in Systematic Literature Reviews in Software Engineering". IEEE Access 8 (2020): 36226-36243.

12. Haddaway NR., et al. "The Role of Google Scholar in Evidence Reviews and Its Applicability to Grey Literature Searching". PLOS ONE 10.9 (2015): e0138237.

13. Piasecki J., et al. "Google Search as an Additional Source in Systematic Reviews". Science and Engineering Ethics 24.2 (2018): 809-810. 
14. Bramer WM., et al. "A Systematic Approach to Searching: An Efficient and Complete Method to Develop Literature Searches". Journal of the Medical Library Association 106.4 (2018): 531-541.

15. Mastrangelo G., et al. "Literature Search on Risk Factors for Sarcoma: PubMed and Google Scholar may be Complementary Sources". BMC Research Notes 3.1 (2010): 131.

16. Falagas ME., et al. "Comparison of PubMed, Scopus, Web of Science, and Google Scholar: Strengths and Weaknesses". FASEB Journal 22.2 (2008): 338-342.

17. Shultz M. "Comparing Test Searches in PubMed and Google Scholar". Journal of the Medical Library Association 95.4 (2007): 442-445.

18. Teng CL. "In Search Of Malaysia: Pubmed, Google Scholar Or Scopus?" IeJSME 2.2 (2008): 5-8.

19. Xue J., et al. "Searches Comparing between Google Scholar and PubMed”. Library Information Services 52.07 (2008): 133-135.

20. Freeman MK., et al. "Google Scholar versus PubMed in Locating Primary Literature to Answer Drug-Related Questions". Annals of Pharmacotherapy 43.3 (2009): 478-484.

21. Wang D. "Comparison of the Strengths and Weaknesses of PubMed, Scopus, Web of Science and Google Scholar". Journal of Agriculture Research and Life Sciences 22.2 (2010): 109-112.

22. Anders ME and Evans DP. "Comparison of PubMed and Google Scholar Literature Searches". Respiratory Care 55.5 (2010): 578-583.

23. Hightower $\mathrm{C}$ and Caldwell C. "Shifting Sands: Science Researchers on Google Scholar, Web of Science, and PubMed, with Implications for Library Collections Budgets". Issues in Science and Technology Librarianship (2010).

24. Tober M. "PubMed, ScienceDirect, Scopus or Google Scholar - Which is the Best Search Engine for an Effective Literature Research in Laser Medicine?" Medical Laser Application 26.3 (2011):139-144.

25. Nourbakhsh E., et al. "Medical Literature Searches: A Comparison of PubMed and Google Scholar". Health Information and Libraries Journal 29.3 (2012): 214-222.

26. Perryman C. "For Non-Expert Clinical Searches, Google Scholar Results are Older with Higher Impact While PubMed Results Offer More Breadth". Evidence Based Library and Information Practice 8.2 (2013): 254-257.
27. Shariff SZ., et al. "Retrieving Clinical Evidence: A Comparison of PubMed and Google Scholar for Quick Clinical Searches". Journal of Medical Internet Research 15.8 (2013): e164.

28. Bramer WM., et al. "The Comparative Recall of Google Scholar Versus PubMed in Identical Searches for Biomedical Systematic Reviews: A Review of Searches Used in Systematic Reviews". Systematic Reviews 2.1 (2013): 115.

29. Wakimoto DK. “Google Scholar Retrieves Twice As Many Relevant Citations As PubMed and Provides Greater Full-Text Access for Quick, Clinical Nephrology Searches". Evidence Based Library and Information Practice 9.1 (2014): 36-38.

30. Badgett RG., et al. "An Experimental Search Strategy Retrieves More Precise Results than PubMed and Google for Questions about Medical Interventions". PeerJ 3 (2015): e913.

31. Gusenbauer M and Haddaway NR. "Which Academic Search Systems are Suitable for Systematic Reviews or Meta-Analyses? Evaluating Retrieval Qualities of Google Scholar, PubMed, and 26 Other Resources". Research Synthesis Methods 11.2 (2020): 181-217.

32. Morshed T and Hayden S. "Google Versus PubMed: Comparison of Google and PubMed's Search Tools for Answering Clinical Questions in the Emergency Department". Annals of Emergency Medicine 75.3 (2020): 408-415.

33. Martín-Martín A., et al. "Google Scholar, Web of Science, and Scopus: A Systematic Comparison of Citations in 252 Subject Categories". Journal of Informetrics 12.4 (2018): 1160-1177.

34. Dunn G. "Design and Analysis of Reliability Studies". Statistical Methods in Medical Research 1.2 (1992): 123-157.

35. Xing X., et al. "Exposing Inconsistent Web Search Results with Bobble". In: Faloutsos M, Kuzmanovic A, editors. Passive and Active Measurement. Springer International Publishing (2014): 131-140.

\section{Assets from publication with us}

- Prompt Acknowledgement after receiving the article

- Thorough Double blinded peer review

- Rapid Publication

- Issue of Publication Certificate

- High visibility of your Published work

Website: www.actascientific.com/

Submit Article: www.actascientific.com/submission.php

Email us: editor@actascientific.com

Contact us: +919182824667 